LAURA EBOLI, Ph.D. ${ }^{1}$

(Corresponding author)

E-mail: laura.eboli@unical.it

GIUSEPPE GUIDO, Ph.D. ${ }^{1}$

E-mail: giuseppe.guido@unical.it

GABRIELLA MAZZULLA, Ph.D. ${ }^{1}$

E-mail: gabriella.mazzulla@unical.it

GIUSEPPE PUNGILLO, Ph.D. student ${ }^{1}$

E-mail: giuseppe.pungillo@unical.it

RICCARDO PUNGILLO, Graduate student ${ }^{1}$

E-mail: riccardopungillo@libero.it

${ }^{1}$ Department of Civil Engineering,

University of Calabria

Via P. Bucci cubo 46/B 87036 Rende (CS), Italy
Traffic Engineering

Preliminary Communication

Submitted: 1 Apr. 2016

Accepted: 16 Nov. 2016

\title{
INVESTIGATING CAR USERS' DRIVING BEHAVIOUR THROUGH SPEED ANALYSIS
}

\begin{abstract}
Speed has been identified for a long time as a key risk factor in road traffic: inappropriate speeds contribute to a relevant part of traffic accidents. Many literature studies have focused on the relationship between speed and accident risk. Starting from this consideration this paper investigates traffic accident risk by analysing the travelling speeds recorded by real tests on the road. A survey was conduct ed to collect experimental speed values in a real context. $A$ specific road segment, belonging to an Italian rural two-lane road, was repeatedly run by 27 drivers in order to collect the instantaneous speed values for each trajectory. Smartphone-equipped vehicles were used to record continuous speed data. The recorded data were used to calculate: the average speed, $50^{\text {th }}$ and $85^{\text {th }}$ percentile speed for each geometric element of the analysed road segment. The main result of the research is the classification of car users' driving behaviour based on the speed values. By using the above mentioned ranges of speed, the classification provides three types of driving behaviour: safe, unsafe, and safe but potentially dangerous. It was found that only four drivers feature "safe" behaviour, driving in a safe manner on most of the road elements. However, the major part of drivers, even if they feature safe behaviour, could be dangerous for other drivers because they drive at very low speeds.
\end{abstract}

\section{KEY WORDS}

road safety; driving behaviour; speed analysis; instantaneous speed values;

\section{INTRODUCTION}

Based on the most recent report of the World Health Organization, in the year 2013 about 1.25 million people were killed on the world roads, that is, about 3 thousand people died every day. Road traffic injuries are the eighth leading cause of death globally, and the leading cause of death for young people. The cost of dealing with the consequences of these traffic accidents runs to billions of dollars. The governments of the world declared 2011-2020 as the "Decade of action for road safety"; the goal of the Decade (20112020) being to reduce the increasing trend in road traffic fatalities, saving an estimated 5 million lives over the period [1].

Traffic accidents are generally determined by a combination of more factors related to the components of a system including roads, the environment, vehicles and road users, and the way they interact. For almost all the traffic accidents the main cause is certainly human error. In fact, relative proportion of contributing factors due to the driver behaviour amounts to $93 \%$, while $30 \%$ is the relative proportion linked to the interrelation between roadway and driver factors [2]. Considering this evidence, the road safety concept cannot be separated from the analysis of human behaviour, the driver being a contributing factor that can be modified; in other words the driving behaviour can be adapted to the road, environment and vehicle conditions. So, traffic accidents and accident severities can be reduced by implementing specific measures to target the driving behaviour [2]. The report of the World Health Organization confirms that among all the risk factors related to the driving behaviour, speeding is considered as the major road safety problem in all countries. There are some studies in the literature investigating speeding as the cause of accident. As an example, the study of Cabral et al. [3] aims to contribute to the reduction of accidents caused by speeding, and, through the use of multisensory information, to help the driver maintain a more regular driving and controlled speed. They focus their 
work on young drivers. On the other hand, the study proposed by Chevalier et al. [4] focused on old drivers' speeding behaviour. They investigated whether a reduction in speeding forms part of the self-restrictive driving behaviour evident when older drivers experience poor cognitive and visual function. Anyway, from the statistics on the road accidents, it emerges that higher driving speeds increase the likelihood of a crash occurring, and the crash severity [2]. Considering this evidence, interventions to reduce speed can surely lead to significant reductions in traffic injuries [1]. For this aim, classifying driving behaviour on specific driving styles can be useful in identifying the preventive measures to be adopted.

In 1990 Reason et al. [5] introduced the Driver Behaviour Questionnaire (DBQ), which consisted of 50 items describing a variety of errors and violations during driving. Since the work of Reason et al. (1990) [5] the popularity of DBQ has increased tremendously, and there exist many variants of DBQ. Several studies investigated the relationship between driving behaviour and road accidents by means of these instruments. The work of Winter and Dodou (2010) [6] analysed 174 studies using DBQ. However, this kind of studies are based only on drivers' statements. On the other hand, there are studies investigating driver behaviour on the basis of kinematic parameters such as speed and accelerations. These studies attempt to classify the driving behaviour and driving style (e.g. Eboli et al. [7]). The driving style is intended as the whole of manoeuvres that a driver performs while driving, in safe or unsafe conditions. Different drivers have different driving styles: driver's personal characteristics and habits will affect their reaction when faced with a dangerous situation while driving [8].

Traditionally, by considering driving behaviour, the drivers were distinguished on the basis of their level of "aggressiveness" (see for example [9]), where an aggressive driving style is a way of affecting any type of manoeuvre on a vehicle in which the driver deliberately behaves in such a manner as to increase the risk of a road accident. Aggressive drivers have a driving style characterized by high speed together with numerous and sudden changes of the instantaneous speed, which cause sudden acceleration and deceleration. This field has been extensively researched by various researchers. The Pennsylvania Department of Transportation defines aggressive driving as "the operation of a motor vehicle in a manner that endangers or is likely to endanger persons or property". McTish et al., 2016 [10] retain that it is necessary to explore and investigate patterns of aggressive driving behaviour as a way of helping identify potential problem drivers and create a sustainable driving behaviour. In their study, five-year crash dataset was analysed to find common factors associated with the aggressive driving. The US National Highway Traffic Safety Administration (NHTSA) defines as aggressive such driving actions referred not only to speeding (exceeding the posted limit or driving too fast for the conditions), but also to other driving actions such as: improper signalling and lane changing (failing to signal intent, using an emergency lane to pass, or passing on the shoulder, cutting into another car's path); tailgating (driving near the back of another car at too close distance); driving in improper lane (travelling too slow in passing lane causing other drivers to perform more frequent lane changes). As a consequence, we could generally say that an aggressive driver has an "unsafe driving behaviour". In fact, as an example, based on national statistics from the NHTSA it is apparent that aggressive driving is highly related to crash fatality rate (McTish et al., 2016 [10]).

On the contrary, some researchers have distinguished between two main categories of drivers, that is, "aggressive" or "cautious" driver (see for example [11]). Cautious driver is often described also as "careful" (see for example [12]). A driver can be considered as cautious when they try to maintain a constant moderate speed, avoiding sudden acceleration and braking, indicating that they feature "safe driving behaviour".

Taubman - Ben-Ari et al. [13] suggested four broad driving styles: (1) the reckless and careless style, which refers to deliberate violations of safe driving norms and to thrill seeking while driving, and it is characterized by high speed, racing, and illegal passing; (2) the anxious style, which relates to feelings of alertness and tension, along with ineffective relaxation activities when driving; (3) the angry and hostile style, which refers to expressions of irritation and rage, as well as hostile attitudes and acts on the road, and is typified by a tendency for aggressive behaviour, such as cursing or flashing lights at other drivers; and (d) the patient and careful style, which reflects a well-adjusted style of driving, including planning ahead, attention to the road, patience, courtesy, calmness, and obedience to traffic regulations.

The classification of the driving style is an issue that has recently become of interest for many researchers, since this topic is found to be closely related to the environmental sustainability. In fact, as well known, a careful driving style allows minimization of road accident risk, but also reduction of $\mathrm{CO} 2$ emissions, fuel consumption and the vehicle wear [14]. In addition, as noted by Bonsall et al. [9], the key parameters of traffic simulation models have been derived from theory or informed guesswork rather than from observation of real behaviour of the drivers. Starting from this observation, the aim of this work is to introduce a methodology for classifying the driving style of the car drivers on the basis of real road driving data collected for different drivers. Given the different driving style classifications resulting from the literature review, we have chosen to distinguish between "safe" or "unsafe" 
driving behaviour, which we retain as the most general classification. A "safe" behaviour can be defined as a condition in which the driver is far from risk of accidents; on the contrary, "unsafe" behaviour indicates driving with a high level of accident risk. These two kinds of conditions on the basis of a method based on the use of a kinematic parameter, which is speed.

The next section proposes a brief literature review of studies where road safety was investigated in terms of the travelling speed. The third section proposes a description of the method proposed for classifying the driving behaviour, based on the values of the travelling speed. In the fourth section the results are reported, and finally some conclusions about the work are provided.

\section{ROAD SAFETY AND TRAVELLING SPEED: LITERATURE REVIEW}

According to a study conducted by the European Commission [15] over $80 \%$ of European drivers are convinced that driving at excessive speeds is often a determining factor in the occurrence of traffic accidents. At the same time, many drivers exceed the speed limit: a percentage of users between 40 and $60 \%$ violate speed limits, and about $10-20 \%$ of this percentage exceeds the limit imposed over $10 \mathrm{~km} / \mathrm{h}$ (ESRA, 2016) [16].

There are many factors that can influence the speed adopted by a driver on a specific road section. The choice of speed can be influenced by the psychophysical state of the driver, personal preference, social pressure, characteristics of the vehicle and all types of interactions among them, but also by environmental factors as the weather conditions and the characteristics of the road.

The travelling speed affects both the risk of being involved in a traffic accident and the severity of an accident [17]. Many studies have focused their attention on the relationship between speed and accident risk. An exhaustive review is reported by the Australian Transport Safety Bureau [18] and Aarts and van Schagen [19].

The first significant study that allowed an examination of the relationship between vehicle speeds and crash risk was proposed by Solomon [20]. This study is often quoted to supporting the idea that a strong relationship exists between crash risk and speed variance, in the sense of different speeds in a traffic stream. Solomon found that the involvement rates took the form of a U-shaped curve as a function of deviation from the mean speed, according to indicate a higher probability of an accident for both low and high speeds. A few years later Cirillo [21] published results of a study similar to Solomon's, but undertaken on interstate highways rather than rural highways. Also, the Research
Triangle Institute [22] conducted a similar analysis for crashes. The findings of RTI for state highways were presented in terms of accident involvement rates for categories of deviation from the mean speed, calculated in a similar manner to that of Solomon: speed deviation of crash-involved vehicles from the average appears to be positively related to crash probability, especially for arterial highways and interstates. In every way, both studies found a U-shaped relationship and have demonstrated that crash rates were the lowest for the drivers travelling near the mean speed, and increased with deviations above and below the mean. In conclusion, these three studies provide evidence that both driving faster and slower than the surrounding traffic, increase the risk of crash.

More recent studies, especially those carried out in Australia $[23,18,24]$ that used more accurate speed estimates, indicate that vehicles that drive faster than the average on that road have higher crash rate; vehicles that drive slower, however, were found not to have an increased risk. Speed deviation of crash-involved vehicles from the average appears to be positively related to crash probability, especially for arterial highways and interstates.

Very few studies explore the modelling of personal driving style of vehicle drivers based on the driving parameters. As an example, Miyajima et al. [25] modelled the relationship between following distance and speed to identify driving style performance and classify drivers into groups based on their car-following patterns.

Constantinescu et al. [26] investigated personal driving styles of various vehicle drivers based on several driving parameters collected by real-time vehicle tracking, such as speed, acceleration, braking, mechanical work. Based on PCA (Principal Component Analysis), they suggest 5 categories of "aggressiveness": from non-aggressive to very aggressive.

Hong et al. [27] understood and modelled aggressive driving style by implementing an in-vehicle sensing platform using Smartphone technology. They used these data and applied machine learning to build a driver model that evaluates the drivers' driving styles based on a number of driving-related features.

\section{EXPERIMENT}

Starting from the literature review, we developed the idea of defining the driving style of car drivers based on speed values recorded by real-time vehicle tracking, and introducing types of driving behaviour according to ranges of speed.

Specifically, real road driving was tested by analysing instantaneous speed values surveyed through smartphones with embedded GPS chips. Speed values were recorded by means of an app for smartphones 
which allows the geo-referenced kinematic parameters of the vehicle to be detected.

The experiment involved a real context, that is a part of the Italian National road n.106 "Jonica" (SS106), which is a rural two-lane road $491 \mathrm{~km}$ long connecting Apulia with the Calabrian regions, in Southern Italy. SS106 annually experiences a large number of accidents and for this reason it was named the "death road"; as reported by the Italian Bureau of Statistics [28], in 2009 SS106 was the Italian road with the highest death rate $(0.46$ dead persons per kilometre). The analysed road segment is $10 \mathrm{~km}$ long. It is an interurban, almost flat road with a maximum longitudinal grade equal to $4 \%$. Each lane of the road segment is 3.5 meters wide. It lacks in lighting and, although the road is far from significant urban areas, there are junctions, and not detected or inadequately detected private accesses along the route. From a planimetric point-of-view the road segment presents a very discontinuous pattern, with radii of curvature ranging from 120 to 520 metres and very tortuous path, especially in the southern part.

The road segment consists of 29 elements: 15 tangents and 14 curves. Nevertheless, the analysis was performed on 22 elements, because the first five elements and the last two were excluded from the analysis for lack of surveyed data. Each element was characterized by the length $(L)$, curve radius $(R), 50^{\text {th }}$ and $85^{\text {th }}$ percentile speeds $\left(V_{50}, V_{85}\right)$, average speed $\left(V_{m}\right)$, design speed $\left(V_{d}\right)$, posted limit $\left(V_{L}\right)$ (Table 1$)$. Design speed values were calculated by using the formulation proposed by the Italian National Research Council [29], reported in Equation 1, as a function of the curve radius $\mathrm{R}$ and by considering the physical laws that govern a vehicle's motion with respect to the road geometric characteristics.

$V^{2}(1-0.0015 \cdot R)+0.432 \cdot R \cdot V-50.17 \cdot R=0$

The analysed road segment was repeatedly run by drivers in order to collect the instantaneous speed values for each trajectory. To this aim, vehicles equipped with a GPS-embedded Smartphone were used; a specific app allowed the speed values to be recorded, by adopting a frequency of 1 Hertz, together with the instantaneous vehicle positions, that is, latitude and longitude [30]. Starting from these data, the $50^{\text {th }}$ and $85^{\text {th }}$ percentile speeds (this last one is the operating speed), and the average speed of the sampled drivers were calculated for each road element [31] (Table 1).

Table 1 - Characteristics of the road elements

\begin{tabular}{||c|c|c|c|c|c|c|c|c|c|c||}
\hline \hline$I D$ & Road element & $L[\mathrm{~m}]$ & $R[\mathrm{~m}]$ & $V_{50}[\mathrm{~km} / \mathrm{h}]$ & $V_{m}[\mathrm{~km} / \mathrm{h}]$ & $V_{\text {low }}[\mathrm{km} / \mathrm{h}]$ & $V_{85}[\mathrm{~km} / \mathrm{h}]$ & $V_{d}[\mathrm{~km} / \mathrm{h}]$ & $V_{L}[\mathrm{~km} / \mathrm{h}]$ & $V_{\text {high }}[\mathrm{km} / \mathrm{h}]$ \\
\hline \hline 1 & Tangent & 58 & & 54.45 & 54.03 & 54.03 & 66.39 & 100 & 90 & 66.39 \\
\hline 2 & Curve & 118 & 386 & 54.02 & 54.78 & 54.02 & 63.24 & 93.88 & 90 & 63.24 \\
\hline 3 & Tangent & 55 & & 59.28 & 59.96 & 59.28 & 70.91 & 100 & 90 & 70.91 \\
\hline 5 & Curve & 118 & 120 & 58.39 & 61.74 & 58.39 & 73.77 & 59.72 & 90 & 59.72 \\
\hline 6 & Tangent & 344 & & 64.40 & 66.55 & 64.40 & 79.46 & 100 & 90 & 79.46 \\
\hline 7 & Curve & 352 & 356 & 67.85 & 68.73 & 67.85 & 75.78 & 91.03 & 90 & 75.78 \\
\hline 8 & Curve & 143 & 320 & 69.10 & 69.28 & 69.10 & 78.01 & 87.40 & 90 & 78.01 \\
\hline 9 & Tangent & 299 & & 71.02 & 71.52 & 71.02 & 78.54 & 100 & 90 & 78.54 \\
\hline 10 & Curve & 429 & 410 & 70.32 & 71.22 & 70.32 & 78.49 & 96.07 & 90 & 78.49 \\
\hline 11 & Tangent & 242 & & 77.59 & 80.39 & 77.59 & 91.21 & 100 & 90 & 90.00 \\
\hline 12 & Curve & 513 & 380 & 79.94 & 80.54 & 79.94 & 88.83 & 93.32 & 90 & 88.83 \\
\hline 13 & Tangent & 288 & & 80.51 & 81.43 & 80.51 & 89.55 & 100 & 90 & 89.55 \\
\hline 14 & Curve & 370 & 270 & 81.00 & 82.03 & 81.00 & 90.11 & 81.91 & 90 & 81.91 \\
\hline 15 & Tangent & 331 & & 82.25 & 83.17 & 82.25 & 94.16 & 100 & 90 & 90.00 \\
\hline 16 & Curve & 270 & 435 & 76.72 & 83.17 & 76.72 & 98.79 & 98.27 & 90 & 90.00 \\
\hline 17 & Tangent & 333 & & 78.81 & 83.59 & 78.81 & 100.55 & 100 & 90 & 90.00 \\
\hline 18 & Curve & 118 & 520 & 82.06 & 85.43 & 82.06 & 106.23 & 100 & 90 & 90.00 \\
\hline 19 & Tangent & 687 & & 80.59 & 86.48 & 80.59 & 113.82 & 100 & 90 & 90.00 \\
\hline 20 & Curve & 120 & 480 & 79.44 & 85.31 & 79.44 & 118.62 & 100 & 90 & 90.00 \\
\hline 21 & Tangent & 573 & & 81.47 & 89.66 & 81.47 & 119.08 & 100 & 90 & 90.00 \\
\hline 22 & Curve & 190 & 510 & 81.67 & 90.79 & 81.67 & 118.81 & 100 & 90 & 90.00 \\
\hline \hline
\end{tabular}

Note: $L=$ length; $R=$ radius; $V_{50}=50^{\text {th }}$ percentile speed; $V_{m}=$ average speed; $V_{\text {low }}=$ lowest threshold; $V_{85}=85^{\text {th }}$ percentile speed (operating speed); $V_{d}=$ design speed; $V_{L}=$ posted limit; $V_{\text {high }}=$ highest threshold. 
The experiment consisted in gathering instantaneous data when the path was covered by 27 drivers with different driver behaviour. A total of 27 real tests was made, all covered in the same direction; during the tests they registered 7,818 instantaneous values of vehicle positioning (latitude and longitude), with an average value of 290 points per survey. In Table 2 we reported some characteristics of the drivers. The drivers were prevalently males (23 out of 27), aged from 22 to 60; on the average, the sample is made up of young drivers at an age of 34 . Real road driving was tested by drivers who did not habitually run the road segment: only 9 out of 27 drivers were habitual users of the analysed SS106 road segment. Finally, the tested drivers were distinguished among three levels of driving experience. The level of driving experience was determined by considering the years of driving license and the average number of annual Kms. More specifically, we assigned three levels to an indicator of driving license according to the following criteria: the indicator is equal to 1 if the years of driving license are lower than 5; 2 if the years are between 6 and 10; 3 if the years are more than 10. Analogously, we assigned three levels to an indicator of annual Kms according to the following criteria: the indicator is equal to 1 if the number of Kms is lower than 10,000; 2 if the number of $\mathrm{Kms}$ is between 10,000 and 30,000; 3 if the number of $\mathrm{Kms}$ is more than 30,000 . The indicator of driving experience is obtained by adding the values of the above described indicators, and by assigning a level of experience according to the following criteria: the level of driving experience is low if the sum is 2 ; it is medium if the sum is 3 or 4; it is high if the sum is 5 or 6. By observing Table 2 it can be stated that 3 out of 27 were drivers with a low level of driving experience (11\% of the sample), 15 out of 27 (56\%) drivers had a medium level of experience, and 9 out of 27 (33\%) could be considered as very expert drivers, due to the large number of years since they have the driving license and/or the average number of annual Kms.

\section{CLASSIFICATION OF DRIVING BEHAVIOUR}

The main result of this study is the classification of driving behaviour based on the car driver's travelling speed. The research on driving behaviour focused on the definition of threshold levels and how they relate to the driver performance. The proposed classification provides three types of driving behaviour according to three ranges of speed. The ranges of speed were defined on the basis of the values of the $50^{\text {th }}$ percentile speed $\left(V_{50}\right)$, operating speed $\left(V_{85}\right)$, and average speed $\left(V_{m}\right)$ (calculated by means of the instantaneous recorded speed), the design speed $\left(V_{d}\right)$ and the posted $\operatorname{limit}\left(V_{L}\right)$.
The aim of the methodology is to obtain a judgment on the driving style; instead of having an overall judgment for the entire route, the attempt was made to find a policy that would provide information on the driving behaviour on each geometric element of the analysed road segment. In fact, the methods reported in the literature provide an overall judgment and do not offer the opportunity to provide information to drivers about the features (geometric elements) they need to know to correct their behaviour in order to reduce the errors that can cause fatal effects.

For this purpose, travelling individual speed profiles and speed indicators (threshold values) are necessary, and therefore speed ranges to classify the driving style for each road element (curve, tangent). In particular, two values were defined as threshold: the lowest value and the highest one.

According to the studies on the relationship between the risk and/or the severity of the road accidents and the travelling speed, the lowest threshold is defined as the minimum value between the V50 and the average speed of the sampled drivers. If the speed profile has normal distribution, that is, the kurtosis is zero, the two speed values are just the same.

Many international studies [32, 33] agree in considering the operating speed (V85) as an average speed value which is adequate to the characteristics of the road section; above this value the driving behaviour can be considered as "dangerous". This speed, in fact, is used in many countries as a reference value to establish speed limits. This approach, despite enabling the establishment of credible limits for drivers, in some cases this is not very applicable. As an example, on the most of Italian roads it is very common that the $85^{\text {th }}$ percentile speed is higher than the design speed, which is defined as "the maximum constant speed that an isolated vehicle may maintain, in a safe condition, over a specified section of highway". For this reason, the minimum value between the operating speed, design speed and speed limit was chosen as the highest threshold.

Definitively, in this paper the analysis of the data is performed based on the following criteria: (1) V(lowest threshold $)=\min \left(V_{50}, V_{m}\right)$ and (2) $V$ (highest threshold $)=\min \left(V_{85}, V_{d}, V_{L}\right)$.

Above the highest threshold safe conditions fail, and then drivers are considered to have an "unsafe driving behaviour". It is supposed that also below the lowest threshold safe conditions fail, and the user features dangerous driving behaviour because the vehicle travelling at a reduced speed can be an obstacle to other vehicles travelling on the same road segment, especially in the same direction [34]. More specifically, this behaviour is considered as "safe" for the analysed driver, but "potentially dangerous". In fact, travelling at a speed lower than other vehicles can irritate other drivers and encourage them to overtake; this 
behaviour increases the probability of a traffic accident. For this reason, in many countries, some categories of slow vehicles have no access to the fast-flowing roads. Specifically, the Italian rules enforces an economic sanction to drivers who drive at a certain speed that can be dangerous for the road traffic.

By summarizing, we can have the following driving behaviours:

1) safe (but potentially dangerous) driving behaviour if $V \leq V$ (lowest threshold)

2) safe driving behaviour if $V$ (lowest threshold) $<V<V$ (highest threshold)

3) unsafe driving behaviour if $V \geq V$ (highest threshold). These criteria were applied to the experimental data collected for the analysed road segment.

Table 1 shows the geometric characteristics of the road elements together with the values of the lowest and highest speed thresholds. The proposed driving behaviour classification can be applied in a rural two-lane road for which the design speed and posted limit have a value higher than both the $50^{\text {th }}$ percentile speed and the average speed.

When these hypotheses are not verified we can assert that the "unsafe" driving behaviour is not affected only by the driver's personal characteristics and habits but is due also to the planimetric and altimetric road characteristics and an inappropriate road design.

In Table 2 we reported for each driver a classification of the road elements according to the criteria described above; in other words, for each driver, we know the number of road elements with "safe but potentially dangerous driving behaviour", with "safe driving behaviour", and with "unsafe driving behaviour". By considering this count, we can provide a prevalent

Table 2 - Characteristics and driving behaviour of the sampled drivers

\begin{tabular}{|c|c|c|c|c|c|c|c|c|c|c|}
\hline 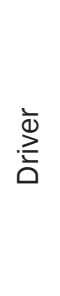 & $\begin{array}{l}\frac{\bar{d}}{0} \\
\frac{\bar{d}}{\bar{d}} \\
0\end{array}$ & $\stackrel{0}{\infty 00}$ & 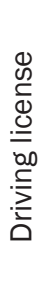 & 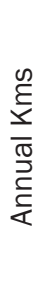 & 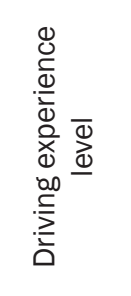 & 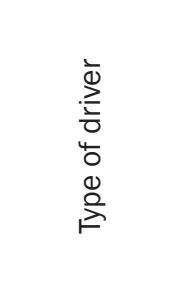 & 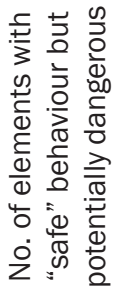 & 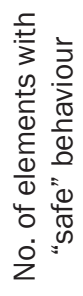 & 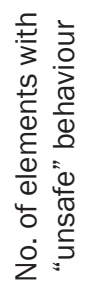 & 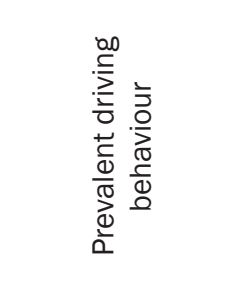 \\
\hline 1 & Male & 27 & 2 & 1 & Medium & Habitual & 4 & 3 & 15 & Unsafe \\
\hline 2 & Male & 27 & 2 & 2 & Medium & Habitual & 8 & 13 & 1 & Safe \\
\hline 3 & Male & 27 & 2 & 1 & Medium & Not habitual & 19 & 2 & 1 & Safe (dangerous) \\
\hline 4 & Male & 27 & 1 & 1 & Low & Not habitual & 10 & 6 & 6 & Safe (dangerous) \\
\hline 5 & Male & 28 & 2 & 1 & Medium & Not habitual & 14 & 5 & 3 & Safe (dangerous) \\
\hline 6 & Male & 28 & 2 & 3 & High & Not habitual & 20 & 2 & 0 & Safe (dangerous) \\
\hline 7 & Male & 29 & 1 & 1 & Low & Not habitual & 0 & 6 & 16 & Unsafe \\
\hline 8 & Female & 28 & 2 & 2 & Medium & Habitual & 16 & 6 & 0 & Safe (dangerous) \\
\hline 9 & Male & 27 & 2 & 2 & Medium & Not habitual & 3 & 8 & 11 & Unsafe \\
\hline 10 & Male & 27 & 2 & 1 & Medium & Not habitual & 0 & 9 & 13 & Unsafe \\
\hline 11 & Male & 35 & 3 & 2 & High & Not habitual & 22 & 0 & 0 & Safe (dangerous) \\
\hline 12 & Female & 49 & 3 & 1 & Medium & Not habitual & 13 & 8 & 1 & Safe (dangerous) \\
\hline 13 & Female & 23 & 1 & 2 & Medium & Habitual & 15 & 6 & 1 & Safe (dangerous) \\
\hline 14 & Male & 55 & 3 & 1 & Medium & Not habitual & 18 & 4 & 0 & Safe (dangerous) \\
\hline 15 & Male & 30 & 2 & 2 & Medium & Not habitual & 13 & 7 & 2 & Safe (dangerous) \\
\hline 16 & Male & 30 & 3 & 2 & High & Habitual & 5 & 9 & 8 & Safe \\
\hline 17 & Male & 27 & 2 & 1 & Medium & Not habitual & 1 & 14 & 7 & Safe \\
\hline 18 & Male & 58 & 3 & 3 & High & Habitual & 11 & 10 & 1 & Safe (dangerous) \\
\hline 19 & Male & 60 & 3 & 3 & High & Not habitual & 17 & 4 & 1 & Safe (dangerous) \\
\hline 20 & Female & 27 & 2 & 3 & High & Not habitual & 2 & 9 & 11 & Unsafe \\
\hline 21 & Male & 58 & 3 & 2 & High & Habitual & 13 & 5 & 4 & Safe (dangerous) \\
\hline 22 & Male & 60 & 3 & 3 & High & Habitual & 10 & 6 & 6 & Safe (dangerous) \\
\hline 23 & Male & 51 & 3 & 2 & High & Habitual & 6 & 14 & 2 & Safe \\
\hline 24 & Male & 26 & 2 & 2 & Medium & Not habitual & 11 & 8 & 3 & Safe (dangerous) \\
\hline 25 & Male & 28 & 2 & 2 & Medium & Not habitual & 16 & 6 & 0 & Safe (dangerous) \\
\hline 26 & Male & 22 & 1 & 1 & Low & Not habitual & 0 & 1 & 21 & Unsafe \\
\hline 27 & Male & 23 & 2 & 1 & Medium & Not habitual & 19 & 2 & 1 & Safe (dangerous) \\
\hline
\end{tabular}


driving behaviour for each driver, on the basis of the highest number of elements including one of the three categories of behaviour. As an example, the first driver is classified as an "unsafe driver" because they prevalently adopted an unsafe behaviour (in 15 out 22 road elements).

By applying the proposed criterion, there are only 4 drivers who feature "safe" behaviour, almost all driving in a safe manner in most of the road elements (about $60 \%$ ); one of these four drivers (driver no. 16) can be considered a borderline case, because they were "safe" in 9 elements, but unsafe in 8 elements (Table 2). All the drivers with a safe driving behaviour are males, and three are young people; they have medium or high driving experience, and they are prevalently habitual drivers of the analysed road. We have calculated the correlations among the different variables reported in Table 2. As an example, we found a discrete negative correlation ( 0.31 is the absolute value) between the type of driver and the number of elements with "safe" behaviour; this means that if the driver is not habitual the behaviour inclines to be unsafe. On the other hand, there are 6 out of 27 drivers who feature "unsafe" behaviour (Table 2). These drivers are prevalently male (5 out of 6 ), and they are all young people; they belong to different categories of driving experience, but they are almost all not habitual users of the analysed road. These results are confirmed by the correlation analysis. In fact, we found a discrete negative correlation ( 0.32 is the absolute value) between age and the number of elements with "unsafe" behaviour; this means that the increase of age entails a decrease of elements with "unsafe" behaviour, so the behaviour inclines to be safe. The same effect is produced by the driving license (with a correlation coefficient equal to -0.44), annual Kms (correlation coefficient of -0.31), and consequently driving experience level (correlation coefficient of -0.41) meaning that if experience level increases, the driving behaviour inclines to be safe. Finally, the category including the drivers with safe but potentially dangerous behaviour is the largest (17 drivers out of 27). Almost all the females (3 out of 4) belong to this category, as well as almost all the less young drivers. We observed a certain positive correlation of age, driving license, driving experience level with the number of elements with "safe but potentially dangerous" behaviour $(0.22,0.30$, and 0.28 , respectively). This means that if age, years of driving license,

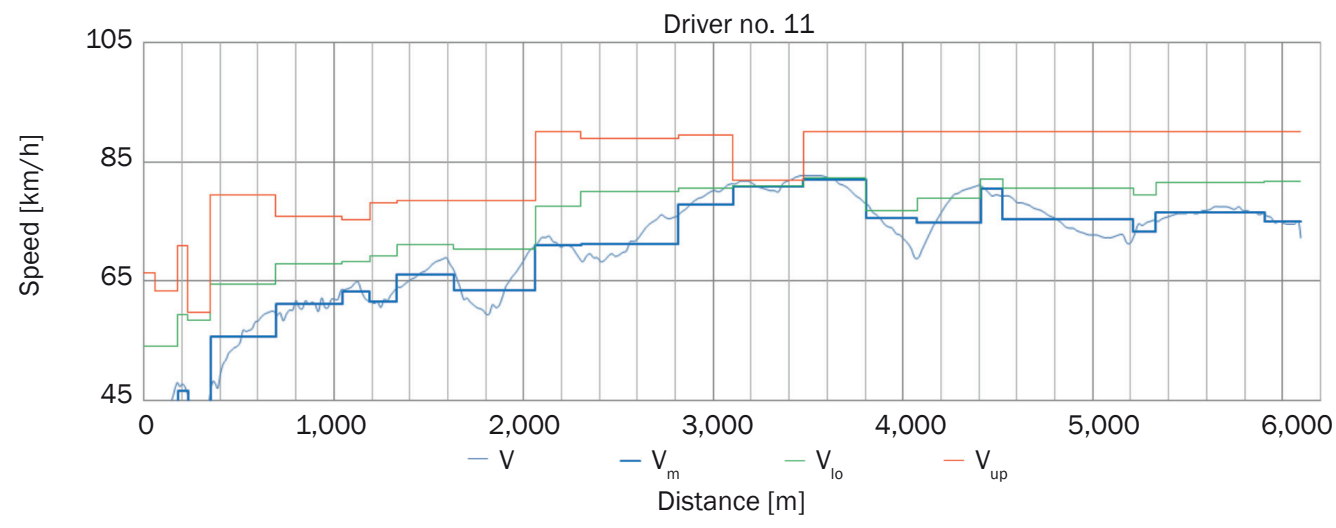

Figure 1 - Example of safe but potentially dangerous driving behaviour

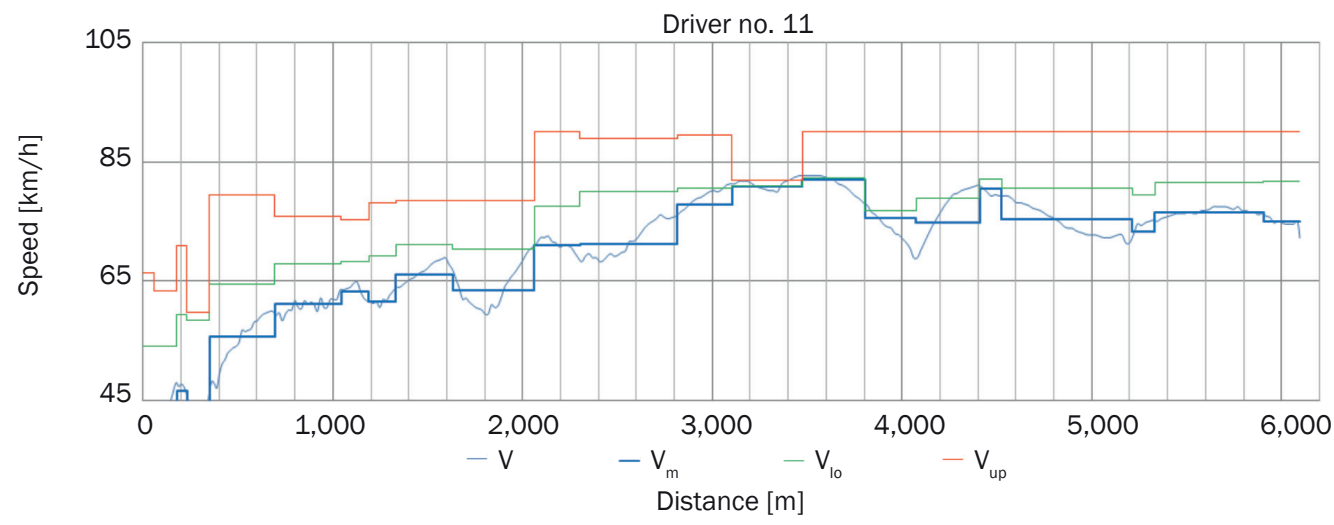

Figure 2 - Example of safe driving behaviour 


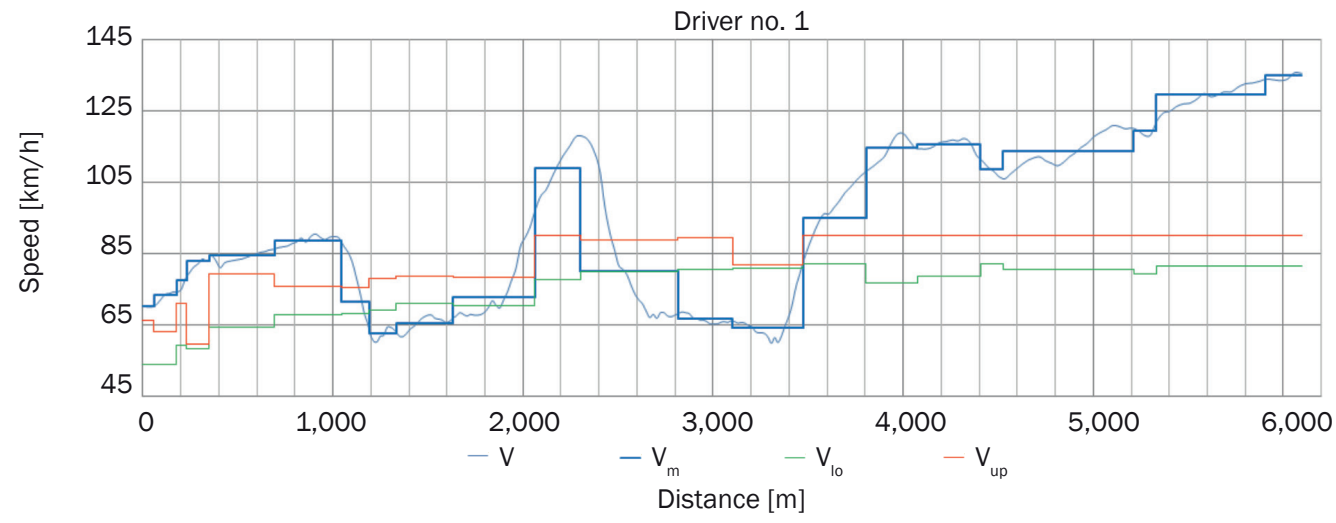

Figure 3 - Example of unsafe driving behaviour

and driving experience increase, the drivers incline to drive at a low speed which can be dangerous for road traffic.

The typical cases are represented by the drivers no. 11, 23 and 1 (Figures 1, 2 and 3). Driver no. 1 can be defined as an "unsafe" driver: in fact, he satisfies the $3^{\text {rd }}$ condition for almost all the road elements. On the contrary, driver no. 23 can be defined as a driver with a "safe" driving behaviour, because in the major part of the road stretch (14 elements out of 22) he satisfies the $2^{\text {nd }}$ condition. Finally, the driver no. 11 can be considered as a safe driver, but potentially dangerous for other drivers, who satisfy the $1^{\text {st }}$ condition in all the road elements.

\section{CONCLUSION}

Driving behaviour includes various driving actions, such as driving above the speed limit, responses to red-light cameras at intersections, behaviour at all-way stops, and attitudes towards pedestrians and cyclists. Out of these, speeding is the most recognized risk factor. The studies suggest that it is particularly difficult to change road-use culture regarding driving speed and observing speed limits [2]. Supporting judicial system plays a role in defining the proper driving behaviour: if drivers know that the speed limits are rarely enforced, drivers will see little reason to reduce their speed. So, in order to reduce traffic accidents, combining different measures seems to offer potential advantages; as an example automated enforcement for speed, appropriate enabling legislation, public education campaigns. Starting from these considerations, we can state that it is very important to investigate the driving behaviour in terms of speed adopted by the drivers. Our paper focused just on this issue. More specifically, we defined the driving behaviour of car drivers based on speed values recorded by real time vehicle tracking, and introducing types of driving behaviour according to ranges of speed. We determined that supporting the research studies about accident risk analysis with real test can help in defining the driving behaviour, also in order to sensitize the drivers about their dangerous driving behaviour and to introduce efficient measures to reduce the travelling speed and traffic accidents.

In addition to the proposed classification of driving behaviour, which can be useful for other researchers for analysing other real contexts, the most relevant findings concern the analysis of driving behaviour in relation to the characteristics of the drivers. We found that if age and driving experience level increase, the behaviour inclines to be safe or the drivers tend to drive at a low speed which can be dangerous for road traffic. However, if the driver is not habitual the behaviour inclines to be unsafe. It can be concluded that young people with low driving experience are more oriented to unsafe driving behaviour in terms of speeding.

Development of the proposed study will surely consist in an extension of the sample in order to better investigate the driving behaviour and the relationship with the drivers' characteristics.

\section{ACKNOWLEDGMENTS}

This work was possible thanks to data collected during P0N01_01541 Project 'M2M mobile to mobility, information and telecommunications system for road safety', which was financed by the Italian Ministry of Education, Universities and Research.

LAURA EBOLI, Ricercatore Universitario, Dottore di Ricerca ${ }^{1}$ E-mail: laura.eboli@unical.it

GIUSEPPE GUIDO, Professore Associato, Dottore di Ricerca ${ }^{1}$ E-mail: giuseppe.guido@unical.it

GABRIELLA MAZZULLA, Professore Associato, Dottore di Ricerca $^{1}$

E-mail: gabriella.mazzulla@unical.it

GIUSEPPE PUNGILLO, Dottorando di ricerca ${ }^{1}$

E-mail: giuseppe.pungillo@unical.it

RICCARDO PUNGILLO, Studente Laurea Magistrale ${ }^{1}$

E-mail: riccardopungillo@libero.it

${ }^{1}$ Dipartimento di Ingegneria Civile, Università della Calabria

Via P. Bucci cubo 46/B Rende (CS) 87036, Italia 


\section{LO STUDIO DEL COMPORTAMENTO DI GUIDA DEI CONDUCENTI DELLE AUTOVETTURE TRAMITE L'ANALISI DELLE VELOCITA'}

\section{SOMMARIO}

La velocità è stata considerata per lungo tempo come un fattore di rischio chiave per il traffico stradale: velocità inadeguate contribuiscono al verificarsi di una parte rilevante degli incidenti stradali. Molti studi in letteratura si sono concentrati sul rapporto tra velocità e rischio di incidente. Partendo da questa considerazione questo lavoro indaga sul rischio di incidente stradale attraverso l'analisi delle velocità registrate tramite prove reali su strada. È stata condotta una indagine per raccogliere $i$ valori di velocità sperimentali in un contesto reale. I conducenti coinvolti nell'indagine (circa 30) hanno percorso ripetutamente un tratto appartenente ad una strada italiana rurale a due corsie, al fine di raccogliere i valori istantanei di velocità per ogni traiettoria. I dati relativi alle velocità sono stati registrati mediante smartphone. I dati registrati sono stati usati per calcolare: la velocità media, il $50^{\circ}$ e l' $85^{\circ}$ percentile delle velocità per ogni elemento geometrico del tratto di strada analizzato. II risultato principale della ricerca è rappresentato da una classificazione del comportamento di guida dei conducenti delle auto, in base ai valori di velocità. La classificazione prevede tre tipi di comportamento di guida: comportamento sicuro, non sicuro, e sicuro ma potenzialmente pericoloso. Si è riscontrato che solo quattro conducenti hanno un comportamento "sicuro", in quanto hanno percorso in modo sicuro la maggior parte degli elementi stradali percorsi. Tuttavia, la maggior parte dei conducenti hanno un comportamento sicuro ma potenzialmente pericoloso per gli altri conducenti, in quanto percorrono il tratto di strada a velocità molto basse.

\section{PAROLE CHIAVE}

sicurezza stradale; comportamento di guida; analisi delle velocità; valori istantanei di velocità;

\section{REFERENCES}

[1] World Health Organization (WHO). Global Status Report on Road Safety; 2015.

[2] American Association of State Highway and Transportation Officials (AASHTO). Highway Safety Manual, first edition; 2009.

[3] Cabral JP, Mendonça RMLO, Cabral JA. Synesthetic Metering for Speed - Evaluation applied to young drivers' speeding. Procedia Manufacturing. 2015;3:61986205.

[4] Chevalier A, Coxon K, Rogers K, Chevalier AJ, Wall J, Brown J, Clarke E, Ivers R, Keay L. A longitudinal investigation of the predictors of older drivers' speeding behaviour. Accid. Anal. Prev. 2016;93:41-47.

[5] Reason J, Manstead A, Stradling S, Baxter J, Campbell K. Errors and violations: a real distinction? Ergonomics. 1990;33:1315-1332.

[6] Winter JCF, Dodou D. The Driver Behaviour Questionnaire as a predictor of accidents: A meta-analysis. Journal of Safety Research. 2010;41:463-470.
[7] Eboli L, Mazzulla G, Pungillo G. Combining speed and acceleration to define car users' safe or unsafe driving behaviour. Transportation Research Part C. 2016;68:113-125.

[8] Chen SW, Fang CY, Ting Tien C. Driving behaviour modelling system based on graph construction. Transp. Res. Part C. 2013;26:314-330.

[9] Bonsall P, Liu R, Young W. Modelling Safety-related Driving Behaviour - Impact of Parameter Values. Transp. Res. Part A. 2005;39(5):425-444.

[10] McTish P, Park S. Exploring aggressive driving behaviour in Pennsylvania's Delaware, Valley region. Procedia Engineering. 2016;145:836-843.

[11] Wang CA, Fu RA, Peng JSB, Mao JA. Driving style classification method for lane change warning system. J. of Transp. Sys. Eng. and Inf. Tech. 2014;14(3):187-193.

[12] Miller G, Taubman-Ben-Ari O. Driving styles among young novice drivers - The contribution of parental driving styles and personal characteristics. Accid. Anal. Prev. 2010;42(2):558-570.

[13] Taubman-Ben-Ari O, Mikulincer M, Gillath O. The multidimensional driving style inventory. Scale construct and validation. Accid. Anal. Prev. 2004;36:323-332.

[14] Van Mierlo J, Maggetto G, Burgwal E, Gense E. Driving Style and Traffic Measures - Influence on Vehicle Emissions and Fuel Consumption. J. Automobile Engineering. 2004;218(1):43-50.

[15] Institut National de Recherchesur les Transports et leurSécurité (INRETS). European drivers and road risk, SARTRE 3 reports. Part 1: report on principal results; 2004.

[16] ESRA (European Survey of Road users' safety Attitudes). Speeding. ESRA thematic Report n.1. 2016. Athens, Greece: National Technical University of Athens.

[17] Institute of Transport Economics (TOI). Speed and road accidents. An evaluation of the Power Model, Report No 740; 2004.

[18] Australian Transport Safety Bureau (ATSB). Travelling speed and the rate of crash involvement on rural roads, Road Accident Research Unit, Report No. CR 204; 2001.

[19] Aarts L, van Schagen I. Driving speed and the risk of road crashes: A review. Accid. Anal. Prev. 2006;38:215224.

[20] Solomon D. Accidents on main rural highways related to speed, driver and vehicle. Washington, DC: US Department of Commerce \& Bureau of Public Roads; 1964.

[21] Cirillo JA. Interstate system crash research; study II, interim report II. Public Roads. 1968;35(3):71-76.

[22] Research Triangle Institute (RTI), Speed and accidents: Volume I, Washington, DC: US Department of Transportation; 1970.

[23] Australian Transport Safety Bureau (ATSB). Travelling speed and the rate of crash involvement, Road Accident Research Unit, Report No. CR 172; 1997.

[24] Australian Transport Safety Bureau (ATSB). Reanalysis of Travelling Speed and the Risk of Crash Involvementin Adelaide South Australia, Road Accident Research Unit, Report No. CR 207; 2002.

[25] Miyajima C, Nishiwaki Y, Ozawa K, Wakita T, Itou K, Takeda K, Itakura F. Driver Modeling Based on Driving 
Behaviour and its Evaluation in Driver Identification. Proceedings of the IEEE. 2007;95(2):427-437.

[26] Constantinescu Z, Marinoiu C, Vladoiu M. Driving Style Analysis Using Data Mining Techniques. Int. J. of Computers, Communications \& Control. 2010;5:654-663.

[27] Hong JH, Margines B, Dey AK. A smartphone-based sensing platform to model aggressive driving behaviours. Proceedings of the SIGCHI Conference on Human Factors in Comput. Syst. Sci. 2014;40474056.

[28] Italian Bureau of Statistics (ACl-Istat). Incidenti stradali in Italia; 2009. Italian

[29] Italian National Research Council (CNR). Norme funzionali e geometriche per la costruzione delle strade. D.M. 5.11.2001 G.U. 4.1; 2002. Italian

[30] Pungillo G. La progettazione e l'adeguamento delle infrastrutture stradali in base alla classificazione di tratti stradali a differente livello di rischio. Dissertation, University of Calabria, Italy; 2013. Italian
[31] Pungillo R. Lo stile di guida dei conducenti delle autovetture: analisi sperimentale della relazione con le velocità di percorrenza. Thesis for the Degree in Civil Engineering, University of Calabria, Italy; 2014. Italian

[32] Camacho-Torregrosa FJ, Pérez-Zuriaga AM, Campoy-Ungría JM, García-García A. New geometric design consistency model based on operating speed profiles for road safety evaluation. Accid. Anal. Prev. 2013;61:33-42.

[33] Majid A, Mehrzad MY. Geometric design consistency model based on speed and safety in rural highways in some European countries: a review. Int. J. of Structural and Civil Engineering Research. 2013;2(2):129-136.

[34] Frith WJ, Patterson TL. Speed variation, absolute speed and their contribution to safety, with special reference to the work of Solomon. Proceedings of the Institution of Professional Engineers New Zealand Annual conference 2001. Auckland, New Zealand. $12^{\text {th }}$ September 2001. 